\title{
Vegetation Database of Successional Permanent Plots in Göttingen
}

\author{
Wolfgang Schmidt, Michaela Dölle \& Andreas Parth
}

\begin{abstract}
Since 1968 an experimental study site on old-field succession in Göttingen (Lower Saxony, Germany) has delivered continuous data on vegetation development on permanent plots. The 23 plots $\left(125-200 \mathrm{~m}^{2}\right)$ cover a range of undisturbed sites since the beginning of the experiment or since 1983 differing in their initial treatment, to plots undergoing a certain yearly treatment like mowing intensities (with and without fertilizer treatment) or ploughing. Beside continuous species lists and cover values, soil chemical properties, biomass data, measurement of PAR or seed bank analysis are available whether continuous or as interval data. Such longterm permanent plot studies, in particular in comparison with similar case studies from all over the world, may enlarge our knowledge on patterns and processes of vegetation dynamics. This report describes the available content in the Vegetation Database of Successional Permanent Plots in Göttingen (GIVD ID EU-DE-015).
\end{abstract}

Keywords: Germany; land abandonment; long-term study; permanent plot.

\section{GIVD Database ID: EU-DE-015}

Last update: 2012-05-09

\section{Vegetation Database of Successional Permanent Plots in Göttingen}

Scope: The database comprises all relevés of a long-term experimental study site on succession on former arable field (Göttingen, Germany). Beside continuous species list and species coverage, soil chemical properties, harvested biomass analysis and seed bank analysis data are available.

Status: completed and continuing Period: 1968-2011

Database manager(s): Wolfgang Schmidt (wschmid1@gwdg.de); Michaela Dölle (mdoelle@gwdg.de); Andreas Parth (aparth@gwdg.de) Owner: University of Göttingen

Web address: http://www.uni-goettingen.de/en/67090.html

Availability: free upon request

Database format(s): MS Access

Online upload: no

Online search: no

Publication: [NA]

Plot type(s): normal plots; time series

Export format(s): [NA]

Non-overlapping plots: 23

Plot-size range: $125-200 \mathrm{~m}^{2}$

Total plot observations: 800

Estimate of existing plots: [NA]

Number of sources: 1

Completeness: [NA]

Valid taxa: 420

Countries: DE: $100.0 \%$

Forest: [NA] — Non-forest: [NA]

Guilds: all vascular plants: $100 \%$; bryophytes (terricolous or aquatic): $10 \%$

Environmental data: altitude: $100 \%$; slope aspect: $100 \%$; slope inclination: $100 \%$; surface cover other than plants (open soil, litter, bare rock etc.) 20\%; soil pH: $20 \%$; other soil attributes: $100 \%$

Performance measure(s): cover: 100\%; measurements like diameter or height of trees: $5 \%$; biomass: $40 \%$

Geographic localisation: GPS coordinates (precision $25 \mathrm{~m}$ or less): $100 \%$

Sampling periods: $1960-1969: 5.0 \%$; 1970-1979: 30.0\%; 1980-1989: 30.0\%; 1990-1999: 20.0\%; 2000-2009: 10.0\%; 2010-2019: 5.0\% Information as of 2012-07-12; further details and future updates available from http://www.givd.info/ID/EU-DE-015

Wolfgang Schmidt (wschmid1@gwdg.de), Michaela Dölle* (mdoelle@gwdg.de), Andreas Parth (aparth@gwdg.de)

Silviculture and Forest Ecology of the Temperate Zones, University of Göttingen, Büsgenweg 1, 37077 Göttingen, GERMANY

*Corresponding author 